\title{
The Expression of Matrix Metalloproteinases in Receptor Activator of Nuclear Factor Kappa-B Ligand (RANKL)-expressing Cancer of Apocrine Origin
}

\author{
YUMI KAMBAYASHI, TAKU FUJIMURA, SADANORI FURUDATE, CHUNBING LYU, \\ TAKANORI HIDAKA, AYA KAKIZAKI, YOTA SATO, KAYO TANITA and SETSUYA AIBA \\ Department of Dermatology, Tohoku University Graduate School of Medicine, Sendai, Japan
}

\begin{abstract}
Primary cutaneous apocrine carcinoma (PCAC) is a rare and highly aggressive tumor entity. Since there is no conventional therapy for advanced PCAC, exploratory treatments are sometimes used. As we previously reported, receptor activator of nuclear factor kappa-B (RANK) ligand $(R A N K L) / R A N K$ signaling on M2 macrophages promotes the production of chemokines and proinflammatory cytokines to maintain the immunosuppressive tumor environment of extramammary Paget's disease (EMPD). Since EMPD is a skin adenocarcinoma of apocrine gland origin that expresses high levels of RANKL and matrix metalloproteinase (MMP) 7, and EMPD is associated with the presence of RANK ${ }^{+} M 2$ macrophages, we hypothesized that tumor-associated macrophages (TAMs) in adenocarcinomas such as PCAC might also express RANKL and MMP7. Materials and Methods: We employed immunohistochemical staining of RANKL and MMP7 in the lesional skin from five patients with $P C A C$, and microarray analysis of MMPs using human monocyte-derived macrophages. Results: According to DNA microarray analysis, the expression of MMP1 and MMP25 was augmented. The DNA microarray results were verified by using real-time polymerase chain reaction (RT-PCR). Immunohistochemical staining of MMP1 and MMP25 as well as chemokine ( $\mathrm{C}-\mathrm{C}$ motif) ligand $(\mathrm{CCL}) 5$ in the lesional skin from five patients with PCAC showed a substantial number of MMP1-bearing cells and MMP25-bearing cells, as well as CCL5-producing cells, that were distributed in the lesional skin. Conclusion: Our study suggests that the RANKL/RANK pathway contributes to the development and maintenance of
\end{abstract}

Correspondence to: Taku Fujimura, Department of Dermatology, Tohoku University Graduate School of Medicine, Seiryo-machi 11, Aoba-ku, Sendai, Miyagi 980-8574, Japan. Tel: +81 227177271, Fax: +81 227177361, e-mail: tfujimura1@ mac.com

Key Words: Primary cutaneous apocrine carcinoma, RANKL, RANK, tumor-associated macrophages, MMPs, denosumab. the immunosuppressive tumor microenvironment and denosumab may be a promising adjuvant therapy targeting TAMs in cancer of apocrine origin.

Primary cutaneous apocrine carcinoma (PCAC) is rare and highly aggressive, being highly metastatic to lymph nodes and other organs, including bone (1-4). Several case reports suggest the successful treatment of metastasized PCAC using chemotherapy with or without radiotherapy (1-4), although drug tolerance is sometimes induced, especially in molecular-targeted therapy (5). Therefore, the induction of an antitumor immune response is important for the long-term response in patients with cancer (6).

Cancer stroma contains various immune cells. Of these, tumor-associated macrophages (TAMs) and regulatory T-cells (Tregs) create an immunosuppressive microenvironment through various pathways. For example, TAMs suppress effector T-cells through immune checkpoint molecules, such as those of the programmed cell death 1 (PD-1) (7) and cytotoxic T-lymphocyte-associated protein 4 (CTLA4) $(8,9)$ pathways. TAMs recruit Tregs by producing CCL17 and CCL22 to maintain the immunosuppressive microenvironment $(8,10-12)$. Since TAMs have been detected both in melanoma and non-melanoma skin cancer $(8,12-14)$, their variable immunosuppressive function is determined by the stimulation from each cancer type and the corresponding stromal factors $(11,13,15,16)$. For example, Paget's cells release soluble receptor activator of nuclear factor kappa-B ligand (sRANKL) into the tumor microenvironment, stimulating RANK $^{+}$TAMs to produce CCL17, which recruits Tregs into the lesional skin of patients with extramammary Paget's disease (EMPD) (11, 17). Accordingly, investigating the characteristics of cancerrelated factors is important to determine the immunological background of the tumor microenvironment in each type of skin cancer.

In the present report, we first investigated the expression of RANKL, matrix metalloproteinase (MMP) 7, CD163, and CD206 in five cases of PCAC and five cases of benign 
apocrine adenoma. Then we analyzed the immunomodulatory effects of sRANKL on TAMs using a DNA microarray database of $\mathrm{CD}_{163}{ }^{+} \mathrm{CD}_{206}{ }^{+} \mathrm{Arg} 1^{+} \mathrm{RANK}^{+} \mathrm{M} 2$ macrophages generated from $\mathrm{CD} 14^{+}$monocytes stimulated by RANKL $(11,18)$. Our results suggest the possible role of RANKL in maintaining the immunosuppressive cancer microenvironment of PCAC.

\section{Materials and Methods}

Reagents. The following antibodies (Abs) were used for immunohistochemical staining: mouse monoclonal Abs for human CD163 (Novocastra, Tokyo, Japan), CD206 (LifeSpan BioScience, Seattle, WA, USA), and CCL5 (LifeSpan BioScience); and rabbit polyclonal Abs for human RANKL (LifeSpan Bioscience), MMP1 (Abnova, Taipei, Taiwan), MMP7 (LifeSpan Bioscience), and MMP25 (Abcam, Tokyo, Japan). The following agents used for cell culture were purchased from Peprotech (London, UK): recombinant human macrophage colony-stimulating factor (M-CSF), human interleukin-4 (IL-4), and sRANKL.

Tissue samples and immunohistochemical staining. We collected archival formalin-fixed paraffin-embedded skin specimens from five patients with PCAC and five patients with benign apocrine adenoma (two cases of apocrine hidrocystoma, two cases of adenoma of the nipple, and one case of syringocystadenoma papilliferum) treated at the Department of Dermatology at Tohoku University Graduate School of Medicine (Table I). The five PCAC samples and five benign apocrine adenoma samples were processed for single staining of RANKL, MMP7, CD163, CD206, MMP1, MMP25 and gross cystic disease fluid protein 15 (GCDFP15), and developed with liquid permanent red (Dako, Tokyo, Japan).

DNA microarray database. As we previously reported, our microarray study data were deposited in the National Center for Biotechnology Information (NCBI) Gene Expression Omnibus and are accessible through GEO Series accession number GSE72292 (18). http:// www.ncbi.nlm.nih.gov/geo/query/acc.cgi?acc=GSE72292.

Culture of M2 macrophages from human peripheral blood monocytes and treatment with or without RANKL. CD14+ cells were isolated from the peripheral blood mononuclear cells (PBMCs) of healthy donors using magnetic-activated cell sorting (MACS) beads (CD14 microbeads, Miltenyi Biotec, Bergisch Gladbach, Germany) according to the manufacturer's protocol. CD14 ${ }^{+}$monocytes $\left(2 \times 10^{5} / \mathrm{ml}\right)$ were cultured in complete medium containing $100 \mathrm{ng} / \mathrm{ml}$ of recombinant human M-CSF for 5 days, as previously reported (11). On the fifth day, the monocyte-derived M2 macrophages (M2M $\phi$ ) were treated with IL-4 $(20 \mathrm{ng} / \mathrm{ml})$ together with or without RANKL $(100 \mathrm{ng} / \mathrm{ml})$ for 6 hours for the analysis of mRNA expression.

RNA extraction, assessment of quality and reverse transcription, and quantitative real-time PCR. Total RNA was extracted using an RNeasy Micro kit (Qiagen, Courtaboeuf, France) in accordance with the manufacturer's instructions. The RNA was eluted with $4 \mu \mathrm{l}$ of RNase-free water. DNase I treatment (RNase-Free DNase Set; Qiagen) was performed to remove contaminating genomic DNA. Reverse transcription was performed by using SuperScript VILO cDNA Synthesis Kit (Invitrogen). Amplification reactions were performed by using an Mx 3000P Real-Time Quantitative PCR System (Stratagene, Tokyo, Japan). The thermal cycling conditions were 3 minutes for polymerase activation at $95^{\circ} \mathrm{C}$, followed by 40 cycles at $95^{\circ} \mathrm{C}$ for 5 seconds and $60^{\circ} \mathrm{C}$ for 20 seconds, and finally products were maintained at $4^{\circ} \mathrm{C}$. Relative mRNA expression levels were calculated for MMP1 and MMP25, and each time point after normalization against glyceraldehyde-3-phosphate dehydrogenase $(G A P D H)$ by using the $\triangle \triangle \mathrm{Ct}$ method. This study was approved by the Ethics Committee of Tohoku University Graduate School of Medicine, Sendai, Japan (2014-1-687) and conducted according to the Declaration of Helsinki principles.

Statistical analysis. For a single comparison of two groups, Student's $t$-test was used. The level of significance was set at $p=0.05$.

\section{Results}

The expression of RANKL and MMP7 on PCAC and benign apocrine adenoma. As we previously reported, Paget cells express RANKL and MMP7 in the lesional skin of a representative skin cancer of apocrine origin, EMPD (11, 17), leading to the recruitment of Tregs through CCL17 produced by TAMs. Therefore, in the present study, we further investigated the expression of RANKL and MMP7 on PCAC and benign apocrine adenoma. We employed immunohistochemical staining for RANKL and MMP7 in five cases of PCAC and five cases of benign apocrine adenoma. As shown in Figure 1, tumor cells expressed RANKL (Figure 1A) and MMP7 (Figure 1B) in all cases of PCAC, whereas only RANKL but not MMP7 was expressed in all cases of benign apocrine adenoma (Figure $1 C$ and D). We summarize the semi-quantitative analysis of immunohistochemical staining in Figure 2.

TAMs in PCAC and benign apocrine adenoma. Since sRANKL stimulates activated $\mathrm{CD} 163^{+} \mathrm{CD} 206^{+}$macrophages to produce a series of chemokines (18), we next evaluated CD163+ macrophages and CD206 ${ }^{+}$cells in the lesional skin of PCAC and benign apocrine adenoma. We immunohistochemically analyzed the expression of CD163 and CD206, which are both markers of tumor-associated M2 macrophages (17). A substantial number of CD163+ macrophages (Figure 3A) and CD206 ${ }^{+}$cells (Figure 3C) were detected in the lesional skin of PCAC. In contrast, only a few $\mathrm{CD} 163^{+}$macrophages (Figure $3 \mathrm{~B}$ ) and CD206 ${ }^{+}$cells (Figure $3 \mathrm{D})$ were observed in the lesional skin of benign apocrine adenoma. The number of $\mathrm{CD} 163^{+}$macrophages and $\mathrm{CD} 206^{+}$ cells are summarized in Figure 2.

mRNA expression of CCL5, MMP1, and MMP25 on CD163 ${ }^{+}$ $\mathrm{CD}_{206^{+}} \mathrm{Argl}^{+} \mathrm{RANK}^{+} \mathrm{M} 2$ macrophages is augmented by stimulation with $s R A N K L$. Since we detected expression of RANKL and MMP7 in the lesional skin of PCAC, we next analyzed the immunomodulatory effects of sRANKL on TAMs using $\mathrm{CD}_{163}{ }^{+} \mathrm{CD}_{206}{ }^{+} \mathrm{Arg} 1^{+} \mathrm{RANK}^{+} \mathrm{M} 2$ 


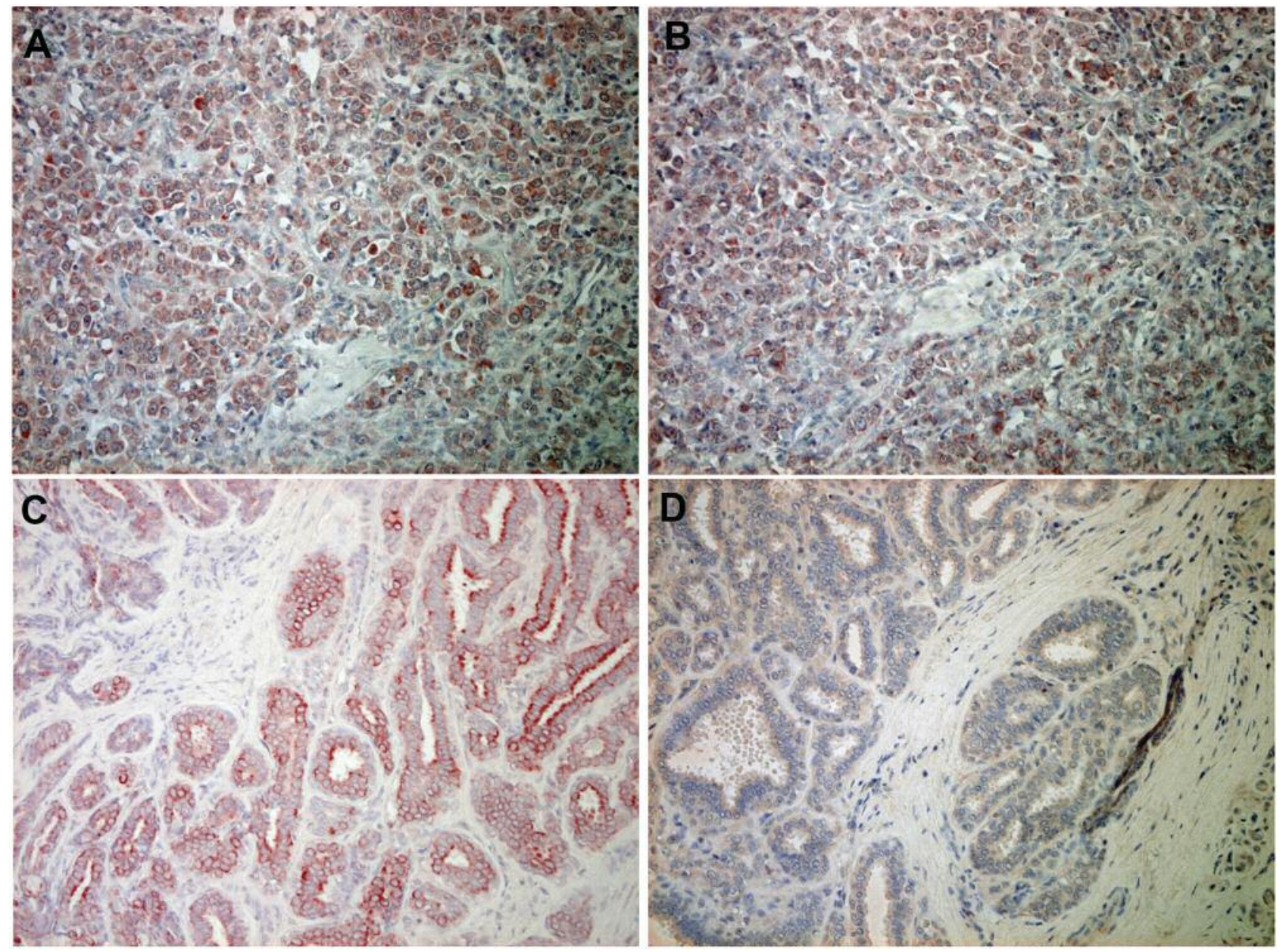

Figure 1. Immunohistochemical staining of receptor activator of nuclear factor kappa-B ligand (RANKL) and matrix metalloproteinase (MMP) 7 on primary cutaneous apocrine carcinoma $(A, B)$ and apocrine hidrocystoma $(C, D)$. Samples were deparaffinized and stained with anti-RANKL $(A, C)$ and anti-MMP7 $(B, D)$. The sections were developed with liquid permanent red. Original magnification: $\times 200$.

macrophages generated from $\mathrm{CD}^{+} 4^{+}$monocytes and a microarray database $(11,18)$. We focused on chemokines and MMP genes that were modified by sRANKL treatment and found that $\mathrm{RANK}^{+} \mathrm{M} 2$ macrophages up-regulated their mRNA expression of CCL5, CCL17, MMP1 and MMP25 after stimulation with sRANKL (http://www.ncbi.nlm. nih.gov/geo/query/acc.cgi?acc=GSE72292.). MMP mRNA expression is depicted in Figure 4A.

To confirm the results of the microarray data, we performed quantitative RT-PCR analysis of monocytederived M2 macrophages that were treated with or without $100 \mathrm{ng} / \mathrm{ml}$ sRANKL (Figure 4B). We focused on the mRNA expression based on the results of the DNA microarray. These results demonstrated that stimulation with sRANKL augmented the expression of $M M P 1$ and $M M P 25$ mRNA by M2 macrophages. Concerning the series of chemokines, the
mRNA expression of CCL5 and CCL17 was confirmed by quantitative RT-PCR, as we previously reported (18).

Expression of MMP1, MMP25, and CCL5 in the lesional skin of PCAC. Since our in vitro study suggested the immunomodulatory effects of sRANKL on $\mathrm{CD} 163^{+} \mathrm{CD} 206^{+}$ macrophages as described above, we hypothesized that the expression of MMP1 and MMP25, as well as CCL5-producing cells, might be observed in the lesional skin of PCAC, which contains abundant $\mathrm{CD} 163^{+} \mathrm{CD} 206^{+}$macrophages. To confirm our hypothesis, we employed immunohistochemical staining of MMP1, MMP25, and CCL5 in five cases of apocrine carcinoma. As expected from the DNA microarray database, a substantial number of both MMP1-bearing mononuclear cells (Figure 5A) and MMP25-bearing mononuclear cells (Figure $5 \mathrm{~B})$ were detected in the lesional skin of PCAC. 

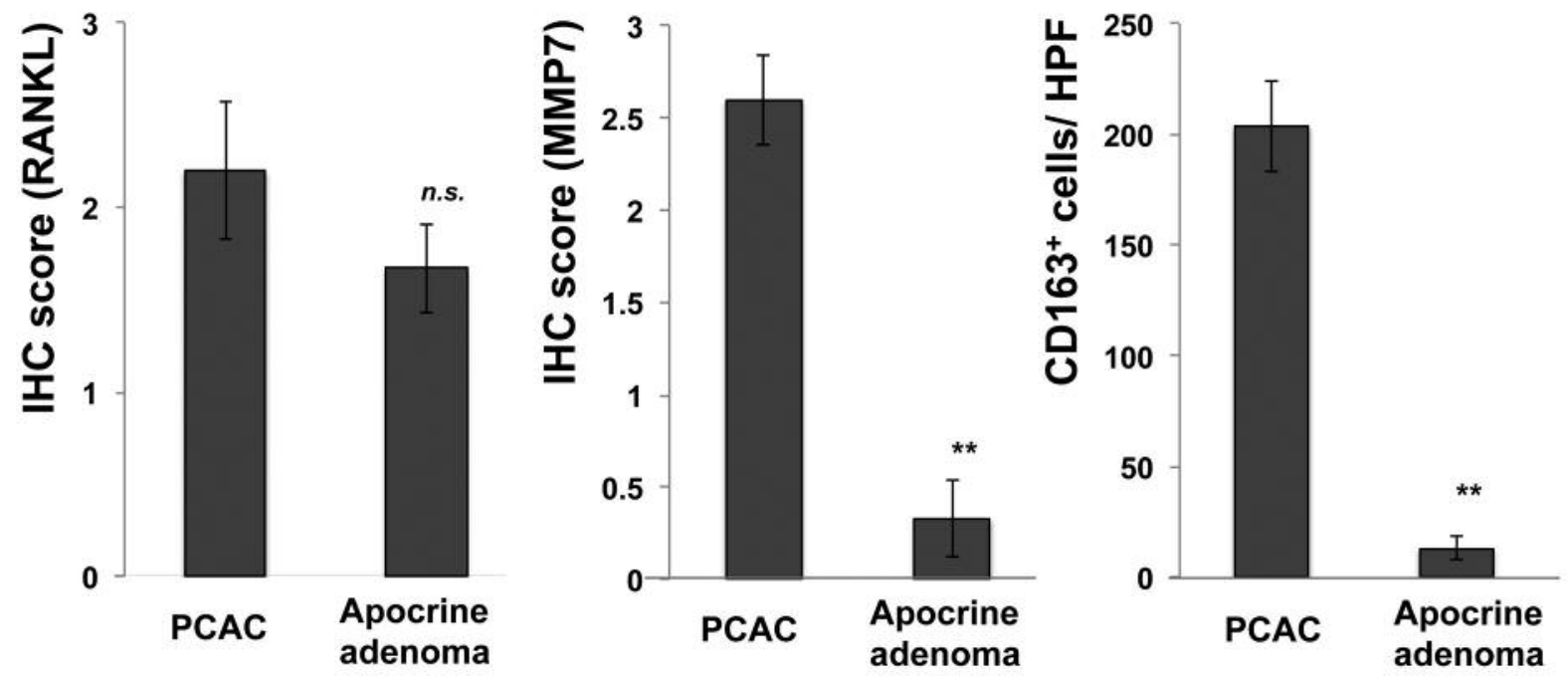

Figure 2. A: The intensity of immunohistochemistry (IHC) staining for receptor activator of nuclear factor kappa-B ligand (RANKL) and matrix metalloproteinase (MMP) 7 for primary cutaneous apocrine carcinoma (PCAC) and apocrine adenoma was scored on a semiquantitative scale. B: Quantification of the numbers of $\mathrm{CD} 163^{+}$cells and $\mathrm{CD}_{206^{+}}$cells in PCAC and apocrine adenoma is shown. Three representative high-power fields of each section were selected from areas of the dermis associated with a dense dermal lymphoid infiltrate. The number of immunoreactive cells was counted using an ocular grid of $1 \mathrm{~cm}^{2}$ at a magnification of $\times 400$. Significantly different at $* * p<0.01$.

\section{Discussion}

The interaction between RANK and its ligand, RANKL, a cytokine member of the tumor necrosis factor superfamily, was first described as the primary mediator of progesteroneinduced proliferation of mammary epithelial cells, and the activation of this pathway promotes mammary tumorigenesis (19). Indeed, Bhatia et al. reported that RANKL is expressed in $90 \%$ of non-neoplastic breast tissue, $62 \%$ of non-metastatic invasive ductal carcinomas, and $31 \%$ of metastatic invasive ductal carcinomas (20). In addition, RANK/RANKL signaling is essential for osteoclast formation, function, and survival, and plays a leading role in bone-related pathologies including bone metastases (21). Concerning its effect on cutaneous immune systems, another report suggested the essential role of RANK/RANKL signaling in dendritic cells for the induction of tolerance in skin (22). Loser et al. reported, ultraviolet irradiation up-regulated the expression of RANKL in keratinocytes, which stimulated RANK expression on Langerhans cells, leading to ultraviolet-induced cutaneous immunosuppression (22).

Concerning skin cancer of apocrine origin, RANKL/ RANK signaling facilitates the production of a Treg-related chemokine, CCL17, from CD163 ${ }^{+} \mathrm{CD} 206^{+} \mathrm{Arg} 1^{+} \mathrm{M} 2$ polarized TAMs in the dermis and $\mathrm{CD}^{+} \mathrm{a}^{+} \mathrm{CD} 209^{+}$ Langerhans cells in the epidermis by the stimulation of sRANKL released from Paget cells in the lesional skin of patients with EMPD $(11,17,23)$. In addition, DNA microarray analysis suggested that sRANKL selectively facilitate the production of CCL5, CCL17, MMP1, and MMP25 from M2 macrophages (18). Indeed, substantial numbers of CCL5-producing cells were detected in the lesional skin of patients with EMPD (17), suggesting that the tumor microenvironment of EMPD is polarized to the immunosuppressive M2/Th2 axis through TAMs (24).

Immunosuppressive TAMs promote an immunosuppressive microenvironment in the tumor-bearing host together with Tregs in various types of skin cancer (8). TAMs are a heterogeneous and plastic population of cells that can be functionally reprogrammed to polarized phenotypes by exposure to cancer-related factors $(8,11,14,17,26)$. One of the most important roles of TAMs is the production of specific chemokines that are determined by the stimulation of stromal factors (9). For example, the cancer stroma of mycosis fungoides contains periostin which stimulates TAMs to produce chemokines related to tumor formation in the lesional skin (15). In addition, another report suggested that the administration of IFN $\alpha$ was able to modify chemokine production by TAMs in the lesional skin of patients with mycosis fungoides, leading to the induction of therapeutic effects (16).

Since MMPs are highlighted as a key marker of M2 macrophages (14), another important role of TAMs is their ability to produce MMPs (8). Indeed, CD163+ TAMs produced high levels of MMP9 and MMP11 in cutaneous squamous cell carcinoma (14). In addition, Kale et al. also reported that 

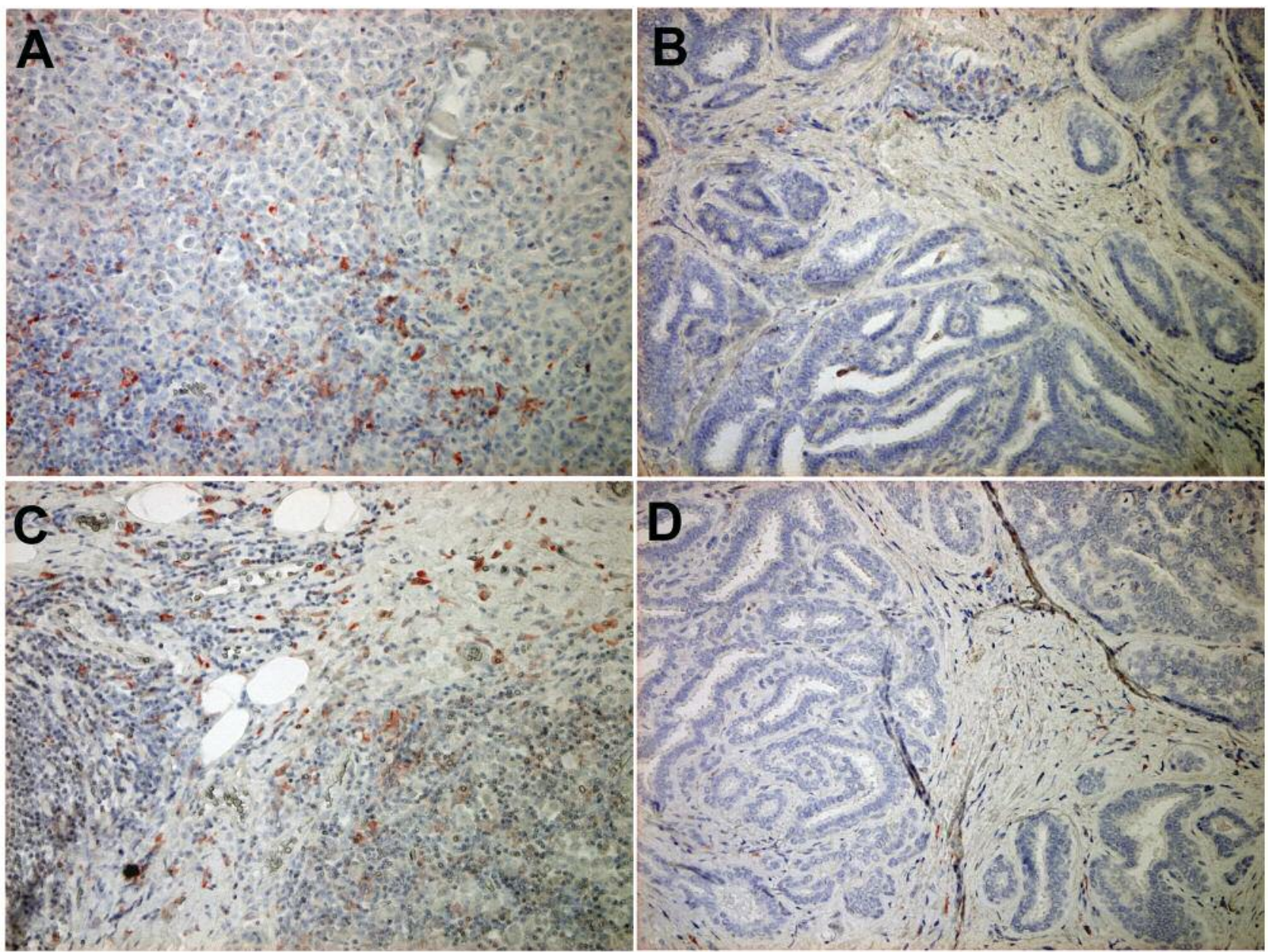

Figure 3. Immunohistochemical staining of CD163 and CD206 on primary cutaneous apocrine carcinoma $(A, B)$ and apocrine hidrocystoma $(C$, $D)$. Samples were deparaffinized and stained with anti-CD163 $(A, C)$ and anti-CD206 $(B, D)$. The sections were developed with liquid permanent red. Original magnification: $\times 200$.

osteopontin signaling in melanoma is involved in macrophage recruitment into tumor by the secretion of MMP9 by TAMs, leading to angiogenesis and tumor growth (27). Concerning RANKL-related MMPs, the expression of both MMP1 and MMP25 correlated to the risk and prognosis of various cancer types $(28,29)$ and was found to be a biomarker of various kinds of cancer (30). As reviewed by Foley and Kuliopulos, MMP1/collagenase-1 degrades type I, type II, and type III collagen to promote tumor invasion (31). Although the exact roles of MMP25 are still unknown, since MMP25 degrades collagens type I to IV, gelatin, fibronectin and fibrin, it might play roles in proteolytic function at the tumor cell surface in the lesional skin of apocrine carcinomas $(29,32)$.

In this report, we first investigated the expression of RANKL and MMP7 on apocrine carcinoma and benign apocrine adenoma. In contrast to the expression of RANKL, the expression of MMP7 was only detected in PCAC. In addition,
TAMs were observed only in PCAC but not in benign apocrine adenoma. Since RANKL stimulates $\mathrm{CD} 163^{+}$monocyte-derived M2 macrophages to increase the mRNA expression of $M M P 1$, $M M P 25$, and CCL5 in vitro, we examined this expression in PCAC. A substantial number of MMP1-bearing cells, MMP25bearing cells, and CCL5-producing cells were detected in the lesional skin of PCAC. In accordance with previous reports, our present study suggests the feasibility of denosumab as an adjuvant therapy for cancer of apocrine origin by targeting TAMs (33). Although PCAC is rare tumor and the number of PCACs was limited in our present report, a previous report described the significance of MMPs in the regulation of breast cancer, highly aggressive apocrine carcinoma, suggesting the significance of MMP1 and MMP25 in the development of PCAC (34). To confirm our hypothesis, further analysis of the mechanisms underlying this phenomenon would offer fundamental insights into the mechanisms involved. 

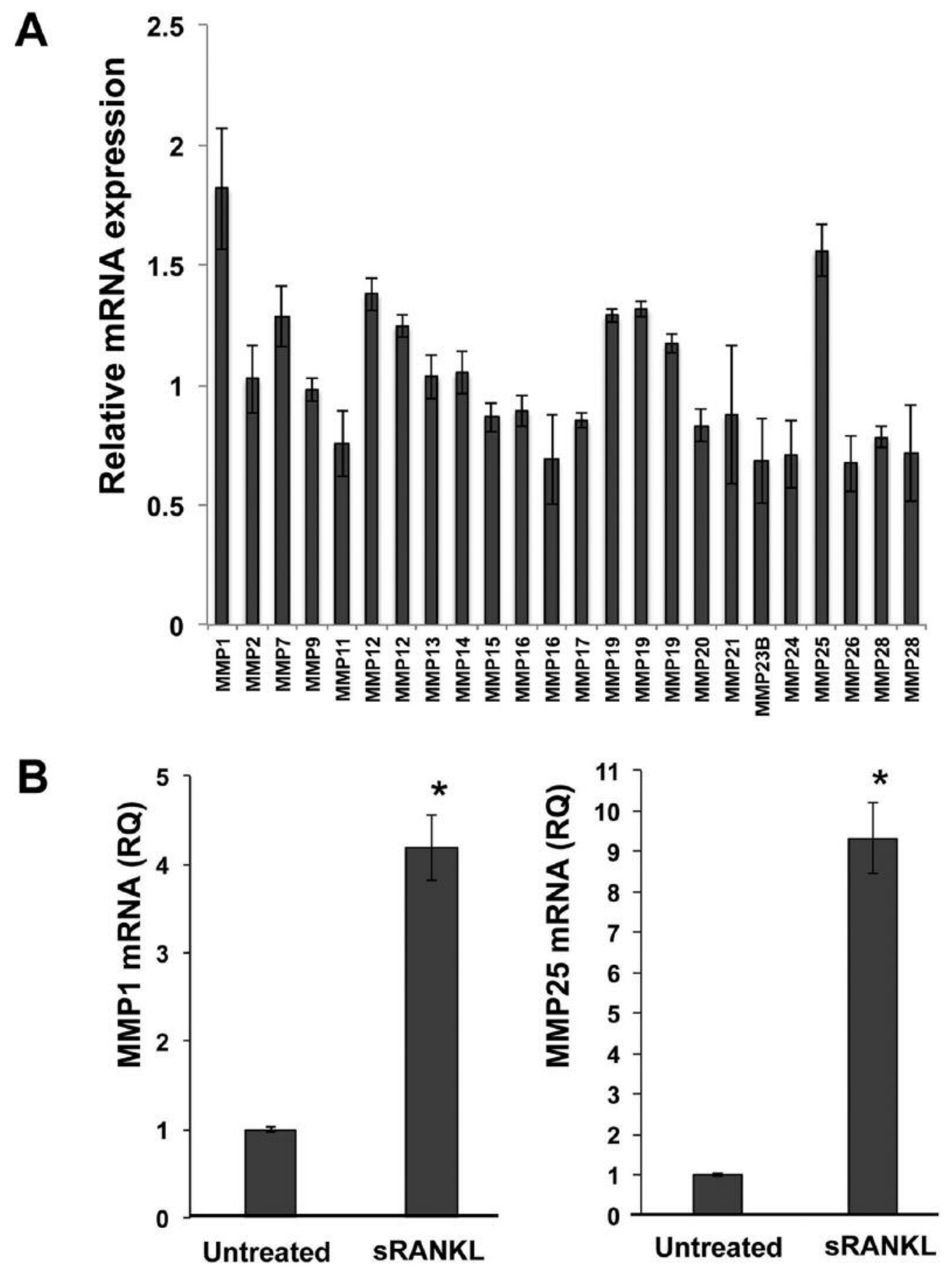

Figure 4. mRNA expression of cytokines in monocyte-derived M2 macrophages stimulated by soluble receptor activator of nuclear factor kappa-B ligand (sRANKL): microarray analysis and quantitative real time polymerase chain reaction. Macrophage colony-stimulating factor/interleukin-4 induced macrophages from peripheral blood mononuclear cells of healthy donors were stimulated with sRANKL (100 ng/ml), as previously described $(8,10)$. Based on gene ontology analysis, we selected a series of cytokines whose mRNA was up-regulated in sRANKL-treated versus untreated cells. Averages of data from three independent experiments are shown (A). The data were deposited in the NCBI Gene Expression Omnibus (http://www.ncbi.nlm.nih.gov/geo/query/acc.cgi?acc=GSE72292). Quantitative real-time polymerase chain reaction for validation of the DNA microarray results was conducted to determine the number of copies of cDNA for each MMP. The mRNA expression levels relative to those of untreated cells were calculated for each gene and time point after normalization against Glyceraldehyde 3-phosphate dehydrogenase (GAPDH) using the $\Delta \Delta^{C t}$ method. The data from each donor were obtained in triplicate assays, and then the mean $\pm S D$ was calculated. Averages of data from at least three independent experiments are shown $(B)$. 

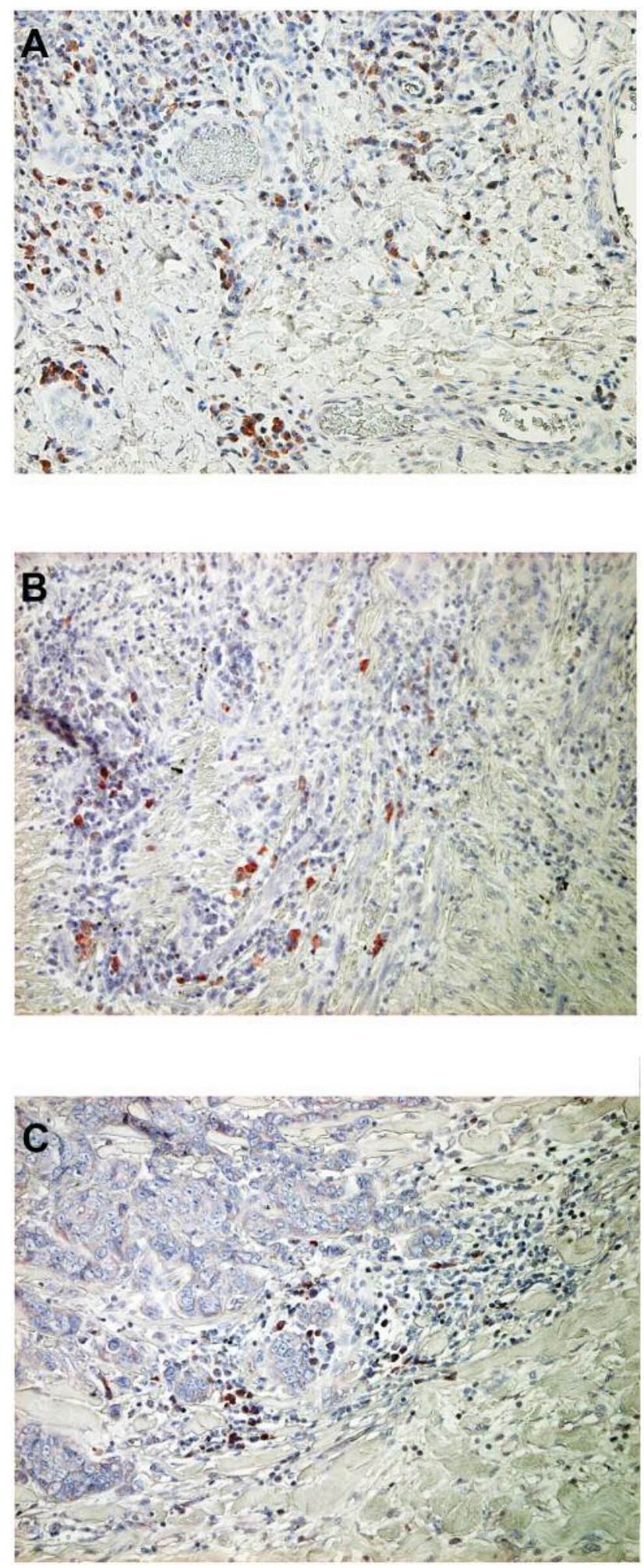

Figure 5. Immunohistochemical staining of matrix metalloproteinase $(M M P) 1, M M P 25$, and chemokine (C-C motif) ligand $(C C L) 5$ on primary cutaneous apocrine carcinoma. Samples were deparaffinized and stained with anti-MMP1 (A), anti-MMP25 (B), and anti-CCL5 $(C)$. The sections were developed with liquid permanent red. Original magnification: $\times 200$.

\section{Conflicts of Interest}

The Authors have no conflicts of interest to declare in regard to this study.

\section{Funding}

This study was supported in part by Grants-in-Aid for scientific research from the Japan Society for the Promotion of Science (K16K155420, K16K101430).

\section{Ethics Approval}

This study was approved by the Ethics Committee of Tohoku University Graduate School of Medicine, Sendai, Japan (2014-1-687).

\section{References}

1 Fujisawa $\mathrm{Y}$ and Fujimoto M: Metastatic cutaneous apocrine carcinoma of the axilla successfully treated using systemic chemotherapy with i.v. epirubicin and cyclophosphamide followed by oral fluorinated pyrimidine.J Dermatol 41: 280-282, 2014.

2 Hidaka T, Fujimura T, Watabe A, Hashimoto A, Haga T, Onami K, Mizuashi M and Aiba S: Successful treatment of HER-2positive metastatic apocrine carcinoma of the skin with lapatinib and capecitabine. Acta Derm Venereol 92: 654-655, 2012.

3 Miller DH, Peterson JL, Buskirk SJ, Vallow LA, Ta R, Joseph R, Krishna M, Ko SJ and Tzou KS: Management of metastatic apocrine hidradenocarcinoma with chemotherapy and radiation. Rare Tumors 7: 6082, 2015.

4 Otsuka M, Yamasaki O, Kaji T, Shien T and Iwatsuki K: Metastatic cutaneous apocrine adenocarcinoma treated with a combination of pertuzumab-based targeted therapy and taxane chemotherapy: a case report. JAMA Dermatol 152: 111-113, 2016.

5 Soura E, Chasapi V and Stratigos AJ: Pharmacologic treatment options for advanced epithelial skin cancer. Expert Opin Pharmacother 16: 1479-1493, 2015.

6 Winkler JK, Schneiderbauer R, Bender C, Sedlaczek O, Fröhling S, Penzel R, Enk A and Hassel JC: Anti-PD-1 therapy in nonmelanoma skin cancer. Br J Dermatol 76: 498-502, 2017.

7 Fujimura T, Ring S, Umansky V, Mahnke K and Enk AH: Regulatory T cells (Treg) stimulate B7-H1 expression in myeloid derived suppressor cells (MDSC) in ret melanomas. J Invest Dermatol 132: 1239-1246, 2012.

8 Fujimura T, Kakizaki A, Furudate S, Kambayashi Y and Aiba S: Tumor-associated macrophages in skin: how to treat their heterogeneity and plasticity. J Dermatol Sci 83: 167-173, 2016.

9 Kalia V, Penny LA, Yuzefpolskiy Y, Baumann FM and Sarkar S: quiescence of memory cd8(+) T-cells is mediated by regulatory T-cells through inhibitory receptor CTLA-4. Immunity 42: 1116$1129,2015$.

10 Adeegbe DO and Nishikawa H: Regulatory T-cells in cancer; Can they be controlled?. Immunotherapy 7: 843-846, 2015.

11 Fujimura T, Kambayashi Y, Furudate S, Asano M, Kakizaki A and Aiba S: Receptor activator of nuclear factor kappa-B ligand (RANKL) promotes the production of CCL17 from RANK+ M2 macrophages. J Invest Dermatol 135: 2884-2887, 2015. 
12 Kakizaki A, Fujimura T, Furudate S, Kambayashi Y, Yamauchi $\mathrm{T}$, Yagita $\mathrm{H}$ and Aiba $\mathrm{S}$ : Immunomodulatory effect of peritumoral administration of interferon-beta on melanoma through tumor-associated macrophages. Oncoimmunology 4: e1047584, 2015.

13 Georgoudaki AM, Prokopec KE, Boura VF, Hellqvist E, Sohn S, Östling J, Dahan R, Harris RA, Rantalainen M, Klevebring D, Sund M, Brage SE, Fuxe J, Rolny C, Li F, Ravetch JV and Karlsson MC: Reprogramming tumor-associated macrophages by antibody targeting inhibits cancer progression and metastasis. Cell Rep 15: 2000-2011, 2016.

14 Pettersen JS, Fuentes-Duculan J, Suárez-Fariñas M, Pierson KC, Pitts-Kiefer A, Fan L, Belkin DA, Wang CQ, Bhuvanendran S, Johnson-Huang LM, Bluth MJ, , Krueger JG, Lowes MA and Carucci JA: Tumor-associated macrophages in the cutaneous SCC microenvironment are heterogeneously activated. J Invest Dermatol 131: 1322-1330, 2011.

15 Furudate S, Fujimura T, Kakizaki A, Kambayashi Y, Asano M, Watabe $\mathrm{A}$ and Aiba S: The possible interaction between periostin expressed by cancer stroma and tumor-associated macrophages in developing mycosis fungoides. Exp Dermatol 25: 107-112, 2016.

16 Furudate S, Fujimura T, Kakizaki A, Hidaka T, Asano M and Aiba S.: Tumor-associated M2 macrophages in mycosis fungoides aquired immunomodulatory function by interferon alpha and interferon gamma. J Dermatol Sci 83: 182-189, 2016.

17 Kambayashi Y, Fujimura T, Furudate S, Asano M, Kakizaki A and Aiba S: The possible interaction between receptor activator of nuclear factor kappa-B ligand (RANKL) expressed by extramammary Paget cells and its ligand on dermal macrophages. J Invest Dermatol 135: 2547-2550, 2015.

18 Fujimura T, Kambayashi Y, Furudate S, Kakizaki A, Hidaka T, Asano $\mathrm{M}$ and Aiba S: Receptor activator of nuclear factor kappaB ligand (RANKL)/RANK signaling promotes cancer-related inflammation through M2 macrophages. Exp Dermatol 25: 397399, 2016.

19 Gonzalez-Suarez E: RANKL inhibition: a promising novel strategy for breast cancer treatment. Clin Transl Oncol 13: 222228, 2011.

20 Bhatia P, Sanders MM and Hansen MF: Expression of receptor activator of nuclear factor-kappaB is inversely correlated with metastatic phenotype in breast carcinoma. Clin Cancer Res 11: 162-165, 2005

21 Boyle WJ, Simonet WS and Lacey DL: Osteoclast differentiation and activation. Nature 423: 337-342, 2003.

22 Loser K, Mehling A, Loeser S, Apelt J, Kuhn A, Grabbe S, Schwarz T, Penninger JM and Beissert S: Epidermal RANKL controls regulatory T-cell numbers via activation of dendritic cells. Nat Med 12: 1372-1379, 2006.

23 Fujimura T, Kambayashi Y, Furudate S, Kakizaki A, Hidaka T and Aiba A: Possible mechanisms of the crosstalk between Langerhans cells and regulatory T-cells in extramammary Paget's disease by receptor activator of nuclear factor kappa-B ligand (RANKL)/RANK pathways. Brit J Dermatol 176: 387$394,2017$.
24 Hagiwara-Takita A, Fujimura T, Kakizaki A and Aiba S: RANKL-expressing extopic extramammary Paget's disease on the lower abdomen. Case Rep Dermatol 8: 130-135, 2016.

25 Zhang Q, Qin J, Zhong L, Gong L, Zhang B, Zhang Y and Gao WQ: CCL5-mediated Th2 immune polarization promotes metastasis in luminal breast cancer. Cancer Res 75: 4312-4321, 2015.

26 Noy R and Pollard JW: Tumor-associated macrophages: from mechanisms to therapy. Immunity 41: 49-61, 2014.

27 Kale S, Raja R, Thorat D, Soundararajan G, Patil TV and Kundu GC: Osteopontin signaling upregulates cyclooxygenase-2 expression in tumor-associated macrophages leading to enhanced angiogenesis and melanoma growth via $\alpha 9 \beta 1$ integrin. Oncogene 33: 2295-2306, 2014.

28 Bradbury PA, Zhai R, Hopkins J, Kulke MH, Heist RS, Singh S, Zhou W, Ma C, Xu W, Asomaning K, Ter-Minassian M, Wang Z, Su L, Christiani DC and Liu G: Matrix metalloproteinase 1, 3 and 12 polymorphisms and esophageal adenocarcinoma risk and prognosis. Carcinogenesis 30: 793-798, 2009.

29 Mäkinen LK, Häyry V, Hagström J, Sorsa T, Passador-Santos F, Keski-Säntti H, Haukka J, Mäkitie AA, Haglund C and Atula T: Matrix metalloproteinase-7 and matrix metalloproteinase-25 in oral tongue squamous cell carcinoma. Head Neck 36: 17831788,2014

30 Stott-Miller M, Houck JR, Lohavanichbutr P, Méndez E, Upton MP, Futran ND, Schwartz SM and Chen: Tumor and salivary matrix metalloproteinase levels are strong diagnostic markers of oral squamous cell carcinoma. Cancer Epidemiol Biomarkers Prev 20: 2628-2636, 2011.

31 Foley CJ and Kuliopulos A: Mouse matrix metalloprotease-1a (Mmp1a) gives new insight into MMP function. J Cell Physiol 229: 1875-1880, 2014.

32 Sun Q, Weber CR, Sohail A, Bernardo MM, Toth M, Zhao H, Turner JR and Fridman R: MMP25 (MT6-MMP) is highly expressed in human colon cancer, promotes tumor growth, and exhibits unique biochemical properties. J Biol Chem 282: 21998-2010, 2007.

33 Azim H and Azim HA, Jr: Targeting RANKL in breast cancer: bone metastasis and beyond. Expert Rev Anticancer Ther 13: 195-201, 2013.

34 Bradbury R, Jiang WG and Cui YX: MDM2 and PSMA play inhibitory roles in metastatic breast cancer cells through regulation of matrix metalloproteinases. Anticancer Res 36: 1143-1151, 2016.
Received October 3, 2017

Revised October 23, 2017

Accepted October 25, 2017 